Research Article

Open Access

\title{
ALK Fusion Protein Expression in Lung Adenocarcinoma and its Correlation with EGFR Protein Expression
}

\author{
Bansi Ladani, Birva Raiya, Nupur Patel and Hemangini H Vora ${ }^{\star}$ \\ Department of Cancer Biology, Immunohaematology Lab, Gujarat Cancer and Research Institute, NCH Compound, Asarwa, \\ Ahmedabad-380 016, India
}

\section{Article Info}

*Corresponding author:
Hemangini H Vora
Associate Professor
Head, Immunohaematology Lab
Department of Cancer Biology
The Gujarat Cancer and Research Institute,
NCH Compound
Asarwa, Ahmedabad-380016
India
Tel: 91792268303
Fax: 917922685490
E-mail: ihcgcri@hotmail.com

Received: October 30, 2018

Accepted: December 5, 2018

Published: December 12, 2018

Citation: Ladani B, Raiya B, Patel N, Vora H. ALK Fusion Protein Expression in Lung Adenocarcinoma and its Correlation with EGFR Protein Expression. Madridge J Oncogenesis. 2018; 2(1): 52-56.

doi: $10.18689 /$ mjo-1000109

Copyright: (c) 2018 The Author(s). This work is licensed under a Creative Commons Attribution 4.0 International License, which permits unrestricted use, distribution, and reproduction in any medium, provided the original work is properly cited.

Published by Madridge Publishers

\begin{abstract}
Aim: To determine ALK fusion protein and EGFR protein expression by Immunohistochemistry in lung adenocarcinoma.

Materials and Method: In this retrospective study, ALK and EGFR expression was evaluated by immunohistochemistry in 101 Formalin Fixed Paraffin Embedded (FFPE) tissues of lung adenocarcinoma.

Results: In this study $11 \%$ patients showed ALK fusion protein expression. In relation to clinical parameters, a higher positive expression was observed in patients with age $<59$, in male gender and tobacco users. Further, with pathological parameters a higher expression of ALK was observed in advanced stage and positive lymph node status. EGFR expression was noted in $72 \%$ patients. In relation to clinical parameters, higher EGFR expression was seen in patients with age $>59$ years, in male gender with tobacco habit. Further, with pathological parameters, a trend of higher EGFR expression was observed in patients with positive lymph node status $(p=0.06)$. In this study $8 \%$ of patients had ALK and EGFR dual expression, which is uncommon.
\end{abstract}

Conclusion: Small group of NSCLC patients harbour ALK rearrangements which are candidate for targeted therapy (Crizotinib). Moreover, co-expression of ALK with EGFR defines a specific subgroup of patients that needs distinct therapeutic strategies.

Keywords: ALK; EGFR; Lung Adenocarcinoma.

\section{Introduction}

Background: Lung carcinoma is the most commonly diagnosed cancer and the leading cause of cancer death worldwide. In spite of continuous efforts to improve the therapeutic response, the overall five-year survival rate for lung cancer is $<15 \%$. This is largely due to its late presentation, chemotherapy resistance, high recurrence rate and lack of curative systemic therapy. Lung cancer ranks among one of the most common and most deadly malignancy. In India the incidence of lung cancer was $6.8 \%$ and at Gujarat Cancer and research Institute, it accounts for 5.8\%.

In era of precision medicine, an assessment of molecular markers such as ALK and EGFR in lung cancer has transformed treatment approach towards targeted therapy. Chromosomal rearrangements in specific "driver genes" are thought to result in oncogenic transformation and tumour growth. This concept moves in the discovery of activating mutations in the epidermal growth factor receptor (EGFR) and rearrangements of the anaplastic large-cell lymphomakinase (ALK). According to literature, the frequency 
of ALK expression in lung adenocarcinoma was observed between 3-7\% [1-4]. Additionally, the prevalence of EGFR expression in Asia was 47.9\% [5] and in Indiaranges between $32-33 \%[6]$.

ALK mutation demonstrate marked sensitivity to treatment with ALK inhibitor (Crizotinib) and EGFR mutation with EGFR inhibitors such as Gefetinib and Erlotinib for longer progression free survival. Epidermal growth factor (EGFR) and Anaplastic lymphoma kinase (ALK) mutation has been considered as mutually exclusive in lung adenocarcinoma, but emerging case reports are available for the presence of this double mutation [7-10].

Earlier, ALK expression in lung carcinoma patients was carried out using FISH and RT-PCR technique. Later, kit of immunohistochemistry was developed for histopathological sub typing of lung carcinoma which gave faster results and was cost effective. Immunohistochemical localisation of CK-7, TTF-1 and CEA markers on tumor tissues have been used to differentiate adenocarcinoma subtypes from other histological subtypes. The purpose of the present study was to evaluate ALK fusion protein status by immunohistochemistry and compared with differential markers and previously studied EGFR expression. Hence, detection of ALK and EGFR protein expression in lung adenocarcinoma patients will be helpful for the selection of targeted therapy in these patients for better outcome.

\section{Materials and Method}

The study was conducted with the approval of Institutional Review Board and Ethics Committee of The Gujarat Cancer and Research Institute. In this retrospective study, 101 lung adenocarcinoma patients were evaluated and the tumour tissue blocks were procured from the archives of the Department of Pathology of the Institute. Other histological subtypes and SERO positive patients were excluded from this study. Detailed clinical history, pathological and radiological findings were recorded. Disease staging was done according to UICC TNM classification.

In this study out of 101 lung adenocarcinoma patients, 79 patients were alive and 22 patients were died within the study period from 2014 to 2018. Moreover, 43 patients were in follow up till study period. Further, 52 patients had received standard chemotherapy and radiotherapy, 18 patients had received Gefetinib along with standard chemotherapy and radiotherapy.

Immunohistochemistry: Immunohistochemistry for ALK and EGFR was performed on formalin fixed paraffin embedded (FFPE) tissue blocks containing primary tumour and Immunohistochemical staining was carried out using Ventana Benchmark XT autoimmuno stainer (Ventana, USA). In this study ALK expression (clone D5F3, Ready to Use, Ventana) was carried out using OptiView DAB detection kit and EGFR (clone EP38Y, Ready to use, Ventana), CK-7, TTF-1 and CEA was carried out using UV DAB detection kit according to manufacturer's protocol. Appendix was used as positive control for ALK (Figure-1) and figure-2 shows negative staining pattern of ALK.

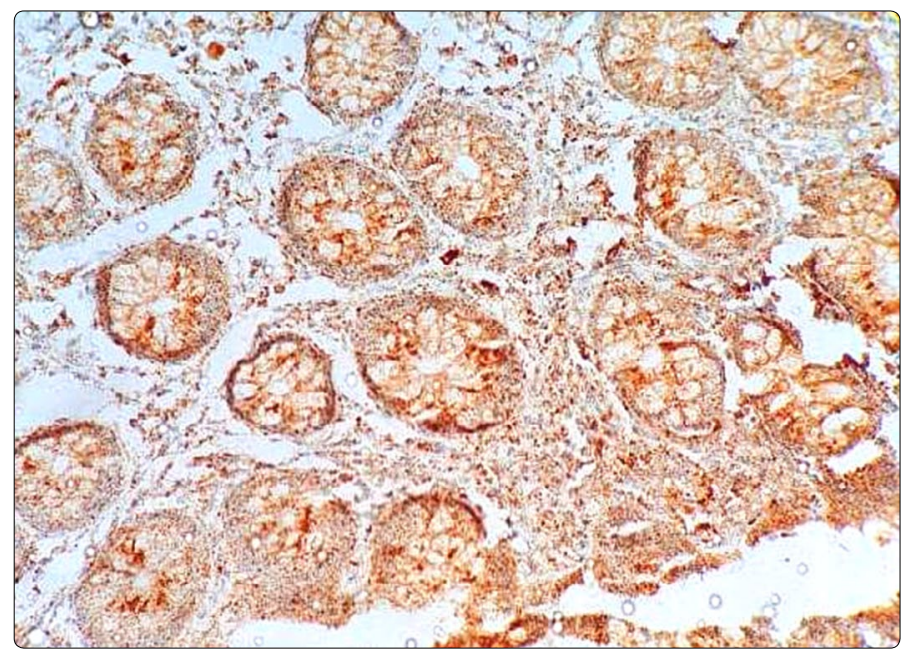

Figure 1. Positive control of ALK (Appendix)

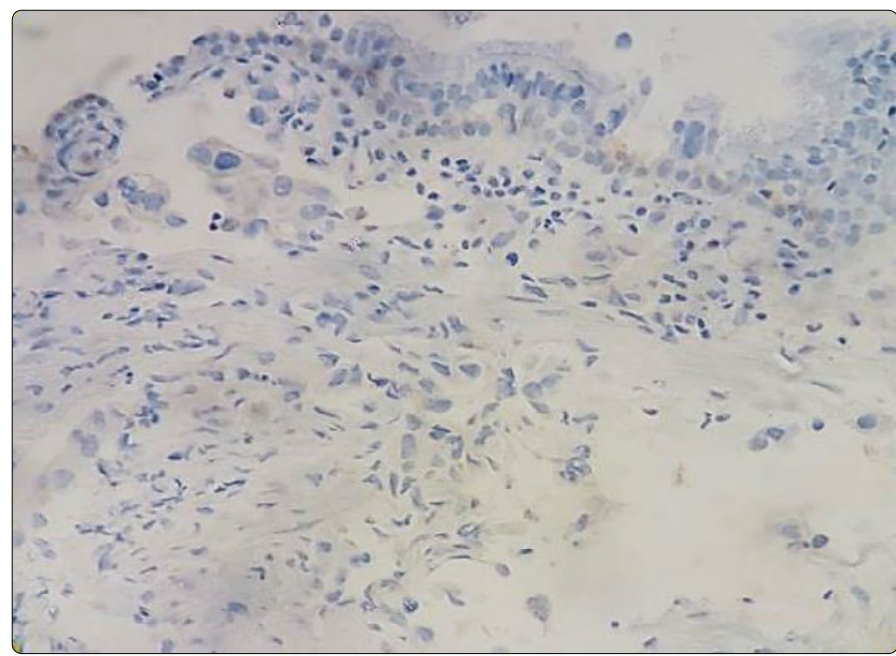

Figure 2. Negative control of ALK

Scoring: Two independent observers familiar with Immunohistochemistry but unaware of the clinical outcome scored all the sections. The tumors were assessed as positive and negative.

Statistical analysis: Statistical analysis was carried out using SPSS statistical software version 20 (SPSS Inc, USA). Mean, standard error (SE) of mean and median were calculated and Pearson's Chi-square test with Pearson's correlation coefficient ( $r$ ) was used to assess correlation and significance between the two parameters. Univariate survival analysis was carried out by Kaplan and Meier method and Log Rank statistics was used to assess the prognostic significance of disease free survival (DFS) and overall survival (OS). p values $\leq$ 0.05 were considered significant.

\section{Results}

\section{ALK fusion protein expression}

In 101 lung adenocarcinoma patients, cytoplasmic staining of ALK expression was observed in $11 \%$ patients (11/101) (Figure-3). 


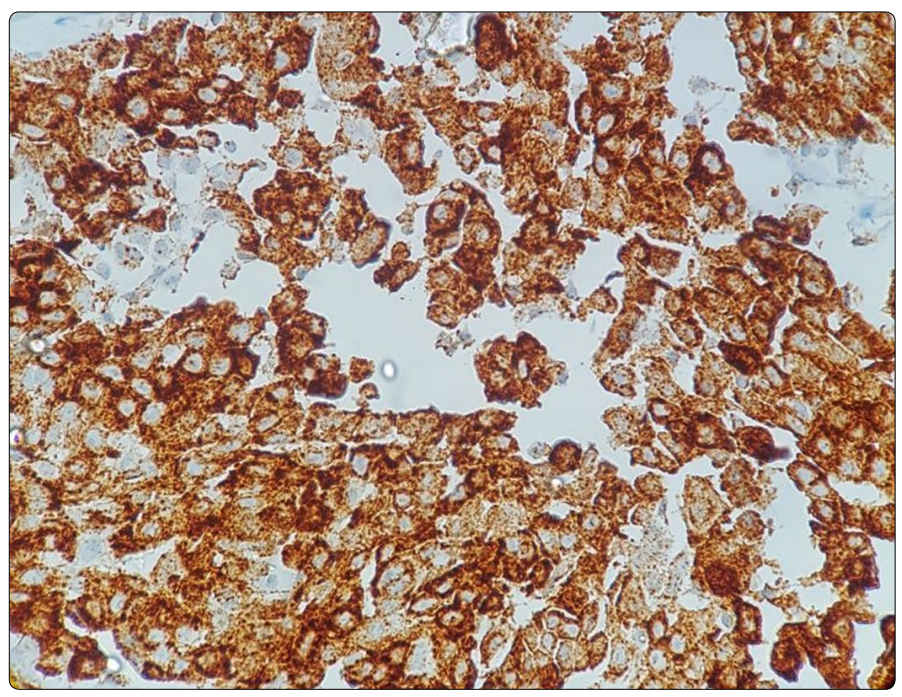

\section{Correlation of ALK Expression with Clinicopathological Parameters}

With respect to clinical parameters, a trend of higher ALK expression was found in younger age population ( $<59$ years). In relation to gender 10 male patients and only 1 female patient exhibited ALK expression. Moreover, ALK expression was seen higher in tobacco habituated patients as compared to their counterparts.

With respect to pathological parameters, ALK expression was seen higher in patients with positive lymph node status and advance stage (stage II, IV) as compared to their counterparts. Among ALK positive patients only 2 patients were developed distant metastasis (Table-1).

Figure 3. Cytoplasmic staining pattern of ALK expression

Table 1. Incidence of ALK and EGFR and its correlation with clinicopathological parameters

\begin{tabular}{|c|c|c|c|c|c|c|c|c|c|c|}
\hline \multirow{2}{*}{ Parameters } & \multicolumn{5}{|c|}{ ALK Expression } & \multicolumn{5}{|c|}{ EGFR Expression } \\
\hline & $\mathrm{N}=101$ & $\begin{array}{c}\text { Negative } \\
\mathrm{N}=90\end{array}$ & Positive $\mathrm{N}=11$ & $x^{2}$ & $p$ value & $N=50$ & $\begin{array}{c}\text { Negative } \\
\mathrm{N}=14\end{array}$ & $\begin{array}{c}\text { Positive } \\
\mathrm{N}=36\end{array}$ & $x^{2}$ & $p$ value \\
\hline \multicolumn{11}{|l|}{ Age (Years) } \\
\hline$\leq 59$ years & $52(52 \%)$ & $45(50)$ & $7(64)$ & \multirow{2}{*}{0.7} & \multirow{2}{*}{0.39} & 21 & $6(43)$ & $15(42)$ & \multirow[b]{2}{*}{0.006} & \multirow[b]{2}{*}{0.93} \\
\hline$>59$ years & $49(48 \%)$ & $45(50)$ & $4(36)$ & & & 29 & $8(57)$ & $21(58)$ & & \\
\hline \multicolumn{11}{|l|}{ Gender } \\
\hline Male & $87(86 \%)$ & $77(85)$ & $10(91)$ & \multirow{2}{*}{0.23} & \multirow{2}{*}{0.62} & 43 & $13(93)$ & $30(83)$ & \multirow[b]{2}{*}{0.75} & \multirow[b]{2}{*}{0.38} \\
\hline Female & $14(14 \%)$ & $13(15)$ & $1(9)$ & & & 7 & $1(7)$ & $6(17)$ & & \\
\hline \multicolumn{11}{|l|}{ Habit } \\
\hline Smokers & $57(56 \%)$ & $51(57)$ & $6(55)$ & \multirow{4}{*}{0.21} & \multirow{4}{*}{0.97} & 25 & $6(43)$ & $19(53)$ & \multirow{4}{*}{2.42} & \multirow{4}{*}{0.29} \\
\hline Chewers & $7(7 \%)$ & $6(7)$ & $1(9)$ & & & 3 & $2(14)$ & $1(3)$ & & \\
\hline Alcohol \& Snuff & $1(1 \%)$ & $1(1)$ & $0(0)$ & & & 0 & 0 & 0 & & \\
\hline None & $36(36 \%)$ & $32(35)$ & $4(36)$ & & & 22 & $6(43)$ & $16(44)$ & & \\
\hline Lymph node & 75 & 67 & 8 & \multirow{5}{*}{0.224} & \multirow{5}{*}{0.97} & 47 & 13 & 34 & \multirow{5}{*}{7.26} & \multirow{5}{*}{0.06} \\
\hline NO & 8 & 7 & 1 & & & 6 & $1(8)$ & $5(15)$ & & \\
\hline N1 & 11 & 10 & 1 & & & 6 & $1(8)$ & $5(15)$ & & \\
\hline N2 & 23 & 21 & 2 & & & 20 & $3(23)$ & $17(50)$ & & \\
\hline N3 & 33 & 29 & 4 & & & 15 & $8(61)$ & $7(20)$ & & \\
\hline TNM stage & 85 & 75 & 10 & \multirow{5}{*}{2.53} & \multirow{5}{*}{0.63} & 50 & 14 & 36 & \multirow{5}{*}{1.10} & \multirow{5}{*}{0.77} \\
\hline Stage I & 2 & $2(3)$ & $0(0)$ & & & 2 & $0(0)$ & $2(6)$ & & \\
\hline Stage II & 10 & $10(13)$ & $0(0)$ & & & 5 & $1(7)$ & $4(11)$ & & \\
\hline Stage III & 19 & $16(21)$ & $3(30)$ & & & 11 & $3(22)$ & $8(22)$ & & \\
\hline Stage IV & 54 & $47(63)$ & $7(70)$ & & & 32 & $10(71)$ & $22(61)$ & & \\
\hline Metastasis & 99 & 88 & 11 & \multirow{4}{*}{2.40} & \multirow{4}{*}{0.30} & 50 & 14 & 36 & \multirow{4}{*}{4.17} & \multirow{4}{*}{0.12} \\
\hline No metastasis & 63 & $54(61)$ & $9(82)$ & & & 26 & $6(50)$ & $20(56)$ & & \\
\hline Distant organ metastasis & 23 & $21(24)$ & $2(18)$ & & & 15 & $3(14)$ & $12(33)$ & & \\
\hline Multiple metastasis & 13 & $13(15)$ & $0(0)$ & & & 9 & $5(36)$ & $4(11)$ & & \\
\hline
\end{tabular}

$\chi^{2}=$ Chi-square, $p$ value $\leq 0.05$ is significant

\section{Univariate survival analysis of ALK}

According to Kaplan Meier, univariate survival analysis, no significant difference in overall survival was noted between ALK negative patients $(23 \%, 18 / 78 ; 7.19 \pm 1.68$ months $)$ and ALK positive patients $(18 \%, 2 / 11 ; 6.90 \pm 2.83$; Log rank=0.59, $d f=1$, $\mathrm{p}=0.44)($ Table-2).

Table 2. Impact of ALK and EGFR expression on overall survival

\begin{tabular}{|c|c|c|c|c|c|c|}
\hline \multirow{2}{*}{ ALK Expression } & No. of patients & $\begin{array}{c}\text { OS in months } \\
\text { Mean } \pm \text { SE }\end{array}$ & $\begin{array}{c}\text { Alive } \\
\text { N (\%) }\end{array}$ & $\begin{array}{c}\text { Dead } \\
\text { N (\%) }\end{array}$ & Log rank & p value \\
\hline Negative & $\mathbf{N = 8 9}$ & $7.19 \pm 1.68$ & $18(23)$ & $60(77)$ & 0.59 & 0.44 \\
\hline Positive & 11 & $6.90 \pm 2.83$ & $2(18)$ & $9(82)$ & \\
\hline \multicolumn{7}{|c|}{ EGFR Expression } \\
\hline Negative & $\mathrm{N}=47$ & & $5(38)$ & $8(62)$ & 0.23 & \\
\hline Positive & 13 & $7.03 \pm 1.15$ & $13(38)$ & $21(62)$ & & \\
\hline
\end{tabular}

$p$ value $\leq 0.05$ is significant, OS: Overall survival 


\section{EGFR expression}

EGFR expression was evaluated in 50 patients. Membranous staining of EGFR expression was noted in $72 \%$ $(36 / 50)$ of patients (Figure-4).

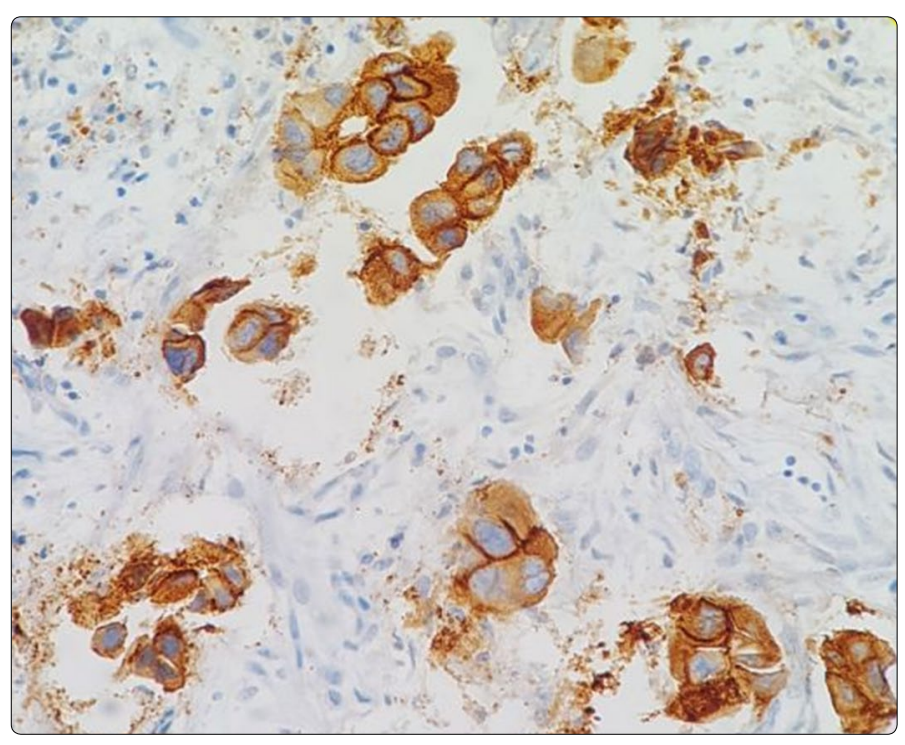

Figure 4. Membranous staining pattern of EGFR expression

\section{Correlation of EGFR with clinicopathological parameters}

With respect to clinical parameters, higher incidence of EGFR expression was observed in patients with older age group (>59 years), male patients and tobacco habituated patients as compared to their counterparts.

With respect to pathological parameters, a trend of higher EGFR expression was observed in patients with N2 lymph node status $(p=0.06)$ and an increasing trend was noted with disease stage (Table-1).

\section{Univariate survival analysis of EGFR}

According to Kaplan-Meier univariate survival analysis, with respect to the overall survival a similar incidence of death and mean months was noted in patients with negative EGFR expression $(38 \%, 5 / 13 ; 7.03 \pm 1.15$ months) and patients with positive EGFR expression (38\%, 13/34; $8.12 \pm 0.96$ months; Log rank=0.23, $d f=1, p=0.62)$ (Table-2).

\section{Correlation of ALK and EGFR with diagnostic adenocarcinoma markers (CK-7, CEA, TTF-1)}

Of 101 patients, details of CK-7, TTF-1 and CEA were available in 91 patients, 92 patients and 62 patients respectively.ALK expression was seen higher in CK-7 positive patients (100\%) followed by CEA positive patients $(87 \%)$ and TTF-1 positive patients (60\%).

Of 50 patients, details of CK-7, TTF-1 and CEA were available in 50 patients, 48 patients and 42 patients respectively. EGFR expression was seen higher in CK-7 positive patients (100\%) followed by CEA positive patients (87\%) and TTF-1 positive patients (79\%) (Table-3).
Table 3. Correlation of ALK and EGFR expression with diagnostic adenocarcinoma markers

\begin{tabular}{|c|c|c|c|c|c|}
\hline \multirow[t]{2}{*}{ Parameters } & \multicolumn{5}{|c|}{ ALK Expression } \\
\hline & $\mathrm{N}(\%)$ & $\begin{array}{c}\text { Negative } \\
\mathrm{N}(\%)\end{array}$ & Positive N (\%) & $x^{2}$ & $p$ value \\
\hline CK-7 & 91 & 81 & 10 & \multirow{3}{*}{0.12} & \multirow{3}{*}{0.65} \\
\hline Negative & 1 & $1(1)$ & $0(0)$ & & \\
\hline Positive & 90 & $80(99)$ & $10(100)$ & & \\
\hline TTF-1 & 92 & 82 & 10 & \multirow{3}{*}{0.001} & \multirow{3}{*}{0.97} \\
\hline Negative & 31 & $27(33)$ & $4(40)$ & & \\
\hline Positive & 61 & $55(67)$ & $6(60)$ & & \\
\hline CEA & 67 & 59 & 8 & \multirow{3}{*}{0.38} & \multirow{3}{*}{0.53} \\
\hline Negative & 14 & $13(22)$ & $1(13)$ & & \\
\hline Positive & 53 & $46(78)$ & $7(87)$ & & \\
\hline EGFR & 50 & 44 & 6 & \multirow{3}{*}{0.096} & \multirow{3}{*}{0.75} \\
\hline Negative & 14 & $12(24)$ & $2(4)$ & & \\
\hline \multirow[t]{2}{*}{ Positive } & 36 & $32(64)$ & $4(8)$ & & \\
\hline & \multicolumn{5}{|c|}{ EGFR Expression } \\
\hline CK-7 & 50 & 14 & 36 & \multirow{3}{*}{-} & \multirow{3}{*}{ - } \\
\hline Negative & 0 & $0(0)$ & $0(0)$ & & \\
\hline Positive & 50 & $14(100)$ & $36(100)$ & & \\
\hline TTF-1 & 48 & 14 & 34 & \multirow{3}{*}{0.35} & \multirow{3}{*}{0.55} \\
\hline Negative & 11 & $4(29)$ & $7(21)$ & & \\
\hline Positive & 37 & $10(71)$ & $27(79)$ & & \\
\hline CEA & 42 & 12 & 30 & \multirow{3}{*}{0.20} & \multirow{3}{*}{0.65} \\
\hline Negative & 5 & $1(8)$ & $4(13)$ & & \\
\hline Positive & 37 & $11(92)$ & $26(87)$ & & \\
\hline
\end{tabular}

$\chi^{2}=$ Chi-square, $p$ value $\leq 0.05$ is significant

\section{Co-expression of ALK and EGFR}

Of 101 patients, small fraction of the cohort $(8 \%, 4 / 50)$ showed dual expression of ALK and EGFR. These patients had stage III and IV disease. Out of them two patients were not received any treatment and lost to follow up (LFU). One patient had received standard chemotherapy (Carboplatin + Pemetrexed) and showed Partial Response (PR), and second patient had received standard chemotherapy along with palliative Radiation therapy (Carboplatin + Pemetrexed + Palliative Radiation therapy) showed disease progression.

\section{Discussion}

The present study aimed to identify anaplastic lymphoma kinase (ALK) fusion protein and epidermal growth factor receptor (EGFR) by immunohistochemistry which is cost effective and rapid technique as compared to FISH and RTPCR technology. In this study ALK expression was observed in $11 \%$ of patients which is high as compared to other studies which shows3-7\% of ALK expression in NSCLC [1-4].

In relation to clinicopathological parameters, ALK expression was found higher in younger age population and in male patients, which was in accordance with other studies $[4,11]$. The present study and study by Martelli et al. [12] observed higher ALK expression in smokers and contrary to that other studies showed a higher ALK expression in nonsmokers or light smokers $[1,4,11,13]$. Furthermore, ALK expression was seen higher in patients with positive lymph node involvement and advanced disease stage. Kamath et al. and Shaw et al. had seen higher ALK expression in patients with metastatic disease $[11,13]$. ALK protein expression and EGFR protein expression was noted in CK-7 and CEA positive patients. 
Regarding EGFR expression, it was seen in $72 \%$ of patients with lung carcinoma and other studies reported EGFR expression in range of $43-89 \%$ in lung adenocarcinoma [1416]. According to the clinicopathological parameters, higher EGFR expression was observed in older age group and in male patients with smoking habit. On the contrary few studies have shown that higher EGFR expression was seen in females and in non-smokers $[7,8,17]$. Further, a trend of higher EGFR expression was found in positive lymph node status and in advance stage disease which was in accordance in study by Lou et al. [8].

EGFR and ALK molecular alteration were found as independent and mutually exclusive cancer driver mutation. The rate of these double mutations is around $1.3 \%$ of nonsmall cell lung carcinoma. In this study $8 \%(4 / 50)$ of patients showed dual ALK expression and EGFR expression. Similarly, some recent studies also observed this co-existence of ALK and EGFR expression in lung adenocarcinoma [7-10]. These patients may need different therapeutic strategy to improve progression free survival.

Differential diagnosis of lung adenocarcinoma is done by immunohistochemical localisation of CK-7, TTF-1 and CEA. Along with these diagnostic markers, use of ALK and EGFR will aid in identifying drug target by cost effective technology and rapid turnaround time.

\section{Conclusion}

In conclusion, a small subset of lung adenocarcinoma exhibit ALK gene rearrangement which express ALK fusion protein. These patients are candidate for ALK inhibitor (Crizotinib). Our study emphasis to test ALK rearrangement also in patients exhibiting EGFR expression by immunohistochemistry because dual ALK and EGFR positive patients may need different therapeutic strategy to better patient's survival. Further, as majority of lung cancer diagnosed at advance stage, ALK and EGFR testing by liquid biopsy which is a non-invasive tool can be used.

\section{Acknowledgments}

We are thankful to Medical Oncology Department, The Gujarat Cancer \& Research Institute, Pfizer and Roche for providing their support to fulfil the present study.

\section{Conflict of Interest}

There is no conflict of interest or no financial benefits regarding this manuscript.

\section{References}

1. Soda $M$, Choi $Y L$, Enomoto $M$, et al. Identification of the transforming EML4-ALK fusion gene in non-small-cell lung cancer. Nature. 2007; 448(7153): 561-566. doi: 10.1038/nature05945

2. Takeuchi $K$, Choi $Y L$, Soda $M$, et al. Multiplex reverse transcription-PCR screening for EML4-ALK fusion transcripts. Clin Cancer Res. 2008; 14(20): 6618-6624. doi: 10.1158/1078-0432.CCR-08-1018

3. Inamura K, Takeuchi K, Togashi $Y$, et al. EML4-ALK fusion is linked to histological characteristics in a subset of lung cancers. $J$ Thorac Oncol. 2008; 3(1): 13-17. doi: 10.1097/JTO.0b013e31815e8b60

4. Wong DW, Leung EL, So KK, et al. The EML4-ALK fusion gene is involved in various histologic types of lung cancers from nonsmokers with wildtype EGFR and KRAS. Cancer. 2009; 115(8): 1723-1733. doi: 10.1002/ cncr.24181

5. Dearden S, Stevens J, Wu, YL, Blowers D. Mutation incidence and coincidence in non small-cell lung cancer: meta-analyses by ethnicity and histology (mutMap). Ann Oncol. 2013; 24(9): 2371-2376. doi: 10.1093/ annonc/mdt205

6. Midha A, Dearden S, McCormack R. EGFR mutation incidence in nonsmall-cell lung cancer of adenocarcinoma histology: a systematic review and global map by ethnicity (mutMapll). Am J Cancer Res. 2015; 5(9): 2892-2911.

7. Yang JJ, Zhang XC, Su J, et al. Lung cancers with concomitant EGFR mutations and ALK rearrangements: diverse responses to EGFR-TKI and crizotinib in relation to diverse receptors phosphorylation. Clin Cancer Res. 2014; 20(5): 1383-1392. doi: 10.1158/1078-0432.CCR-13-0699

8. Lou NN, Zhang XC, Chen HJ, et al. Clinical outcomes of advanced nonsmall-cell lung cancer patients with EGFR mutation, ALK rearrangement and EGFR/ALK co-alterations. Oncotarget. 2016; 7(40): 65185-65195. doi: 10.18632/oncotarget.11218

9. Sweis RF, Thomas $S$, Bank B, et al. Concurrent EGFR mutation and ALK translocation in non-small cell lung cancer. Cureus. 2016; 8(2): e513. doi: 10.7759/cureus513

10. Kamath MP, Lokesh $\mathrm{KN}$, Babu KG, et al. A case report of a metastatic adenocarcinoma of lung with dual positivity for EGFR mutation and ALK fusion. J Nucl Med Radiat Ther. 2015; 6: 262. doi:10.4172/21559619.1000262

11. Shaw AT, Yeap BY, Mino-Kenudson $M$, et al. Clinical features and outcome of patients with non-small-cell lung cancer who harbor EML4-ALK. J Clin Oncol. 2009; 27(26): 4247-4253. doi: 10.1200/JCO.2009.22.6993

12. Martelli MP, Sozzi G, Hernandez L, et al. EML4-ALK rearrangement in nonsmall cell lung cancer and non-tumor lung tissues. Am J Pathol. 2009; 174(2): 661-670. doi: 10.2353/ajpath.2009.080755

13. Rodig SJ, Mino-Kenudson M, Dacic S, et al. Unique clinicopathologic features characterize ALK-rearranged lung adenocarcinoma in the western population. Clin Cancer Res. 2009; 15(16): 5216-5223. doi: 10.1158/1078-0432.CCR-09-0802

14. Gupta R, Dastane AM, Forozan F, et al. Evaluation of EGFR abnormalities in patients with pulmonary adenocarcinoma: the need to test neoplasms with more than one method. Mod Pathol. 2009; 22(1): 128-133. doi: 10.1038/modpathol.2008.182

15. Shigematsu $H$, Lin $L$, Takahashi $T$, et al. Clinical and biological features associated with epidermal growth factor receptor gene mutations in lung cancers. J Nati Cancer Inst. 2005; 97(5): 339-346. doi: 10.1093/jnci/dji055

16. Suzuki $M$, Shigematsu $H$, Hiroshima $K$, et al. Epidermal growth factor receptor expression status in lung cancer correlates with its mutation. Hum Pathol. 2005; 36(10): 1127-1134. doi: 10.1016/j.humpath.2005.08.007

17. Zhang $X$, Zhang $S$, Yang $X$, et al. Fusion of EML4 and ALK is associated with development of lung adenocarcinomas lacking EGFR and KRAS mutations and is correlated with ALK expression. Mol Cancer. 2010; 9: 188. doi: 10.1186/1476-4598-9-188. 